affect the capacity and efficiency of a definite industrial unit. In the second place, it helps the engineer to design new equipment and to predict the performance of a given type of apparatus on any heat transfer or absorption problem from data obtained in operating tests on the same type of machine. The theory is based on the fundamental principles of heat transfer and absorption, and serves to correlate and classify the performance of apparatus designed for these purposes.

\title{
The Colloid Content of Vegetable Tanning Extracts ${ }^{1,2}$
}

\author{
With Attempts to Correlate Astringency with the Potential Difference of the Particles \\ against the Aqueous Phase \\ By Arthur W. Thomas ${ }^{3}$ and Stuart B. Foster \\ Columbia Univgrstyy, New York, N. Y.
}

\begin{abstract}
As recorded in the following paper, measurement of the potential differences of certain vegetable tanning extracts shows that the order of these values is approximately the same as the order of their degrees of astringency. The potential difference increases with dilution of the extract, decreases with rising acidity, and is increased by dialysis of the extract. These simple manipulations suggest possibilities for a more intelligent use of these extracts in vegetable tanning.

Precipitation tests were made on the extracts with various electrolytes. The conduct of the extracts shows that there is a large amount of colloidal matter present, that it belongs to a type of dispersion with properties between the intermediate and hydrophilic dispersions, and, hence, that the measurements reported in the first part of the paper are justified.
\end{abstract}

From the colloidal point of view, vegetable tanning extracts constitute an unexplored field, and the authors believe that the precipitation graphs included in this article will prove of value when interpreted in the light of future research.

A STRINGENT properties of vegetable tanning extracts vary enormously, generally depending upon the source of the extract. If the fundamental reason for this variation could be discovered, rapid progress would be effected in the use of various extracts in vegetable tanning. It would be possible by varying the astringency of a given extract by simple chemical treatment to render an extract suitable for any process, thus avoiding the employment of a given extract or mixture of extracts for making a given kind of leather.

The simple theory of vegetable tanning proposed by Procter and Wilson ${ }^{4}$ is based on a mutual neutralization of the opposite electrical charges of the colloidal tannin particles and the hide colloids. If this theory is correct the electrical charge of the tannin particles should be greater for an astringent extract than for a mild one.

The authors have endeavored to determine the potential difference of the tannin particles in various vegetable extracts against the aqueous phase, $i$. e., the difference in potential of the negative electrical charges on the particles against the positive charges of the ions in the water immediately in contact with the particles.

\section{Apparatus}

The method used for the determination was the U-tube electrophoresis technic as described by Burton. ${ }^{5}$

1 Presented before the Section of Leather Chemistry at the 62nd Meeting of the American Chemical Society, New York, N. Y., September to 10,1921 .

2 Published as Contribution No. 383 from the Chemical Laboratories of Columbia University.

3 Assistant Professor.

1 J. Chem. Soc., 109 (1916), 1327.

" "Physical Properties of Colloidal Solutions," Chapter VII (1916).
The U-tube had limbs about $20 \mathrm{~cm}$. in length and $2 \mathrm{~cm}$. in diameter. The lower half of each limb was graduated in fifths of centimeters, from 0 at the top to $10 \mathrm{~cm}$. at the lower portion. In the bottom of the bend of the U-tube was sealed a delivery tube with stopcock, bent upward and ending above the upper levels of the limbs in a funnel, for the purpose of filling the tube with the solution. The platinum electrodes were sections of cylinders, not spirals as used by Burton.

Before operating, the effective distance through the bend of the tube was measured, so that the potential gradient per centimeter could be calculated. The conductivities of known columns of $0.02 \mathrm{M} \mathrm{KCl}$ solution through the graduated portions of the tube were determined, and the conductivity between the lowest graduated marks on each limb through the bend was compared with these values. The effective distance through the bend was found to be $9.9 \mathrm{~cm}$.

The conductivities of the solutions of all extracts were determined in the usual type of Wheatstone bridge apparatus with a Freas conductivity cell. ${ }^{6}$ Solutions of potassium chloride of the same conductivity were superimposed upon the columns of the extract in the U-tube. The concentrations of chloride required for given conductivities were based upon the figures of Noyes and Falk, ${ }^{7}$ and were obtained by plotting their specific conductance values against concentration of potassium chloride. When the conductance of a tanning solution was obtained the corresponding concentration of potassium chloride was found by merely reading from the curve of Noyes and Falk's data.

All measurements of conductivity and electrophoresis were made at $25^{\circ} \pm 0.005^{\circ} \mathrm{C}$. by submerging the cells and tubes in a large Freas water thermostat which contained $80 \mathrm{gal}$. of water at the temperature stated.

\section{METHOD}

The potassium chloride solution of the same conductivity as the extract to be studied was poured through the funnel and allowed to flow into the bend of the U-tube. The stopcock was closed, the excess chloride remaining in the delivery tube was removed, and the delivery tube was filled with the tanning extract. By gradually opening the stopcock, the extract was allowed to flow very slowly into the bend so that it would not mix with the layer of potassium chloride solution. The final result was a U-tube filled through the bend and up the limbs with extract, with a 2-cm. layer of potassium chloride solution on top of each column. By careful manipulation, the interfaces between colloidal dispersion and electrolyte solution were fairly sharp.

The platinum electrodes were carefully inserted, both to the same level, and submerged in the potassium chloride

- Eimer and Amend, 1920 Catalog, No, 5202.

7 J. A in. Chem. Soc., $34(1912), 454$ 
solution, not coming in contact with the tanning solution. The tube was then immersed in the water bath, and the electrodes were connected with a 115-volt direct house current.

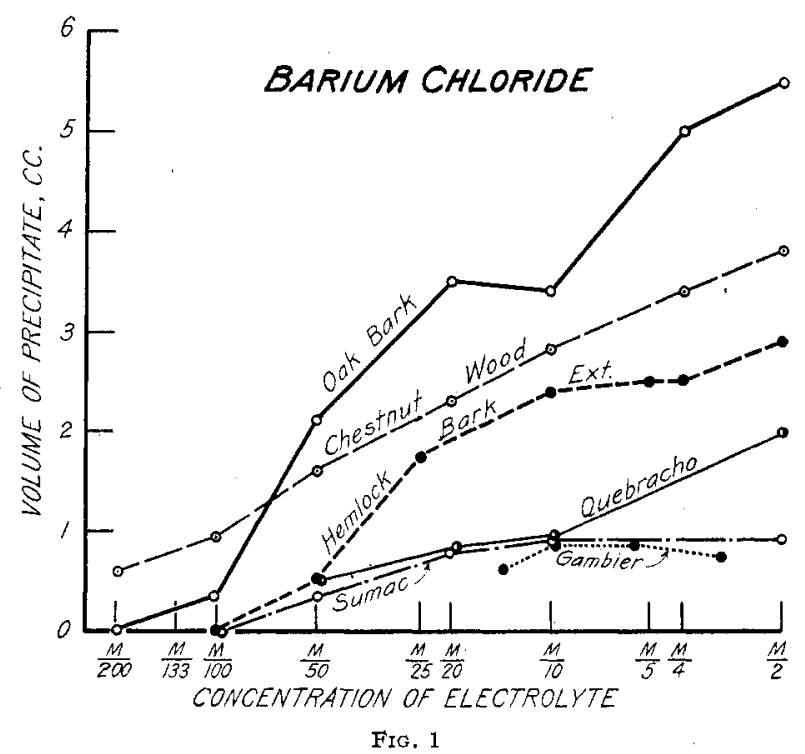

The current was passed for 5 min, reversed, and passed an additional 5 min., in order to flatten the interfacial surfaces. The current was then passed through for $20 \mathrm{~min}$., and the displacement noted during this period. From the values obtained the velocity of migration could be calculated.

The potential differences of the double layers of the particles were calculated by means of the well-known formula of Lamb, ${ }^{8}$ as applied by Burton: ${ }^{9}$

$$
\text { P. D. }=\frac{4 \pi \eta v(300)^{2}}{\mathrm{KX}}
$$

where $P . D .=$ Potential difference in volts.

$\eta=$ Viscosity of dispersion medium (in this case water at $25^{\circ}=10.2 \times 10^{-3}$ )

$v=$ Velocity of migration in $\mathrm{cm}$. per sec.

$\mathrm{K}=$ Dielectric constant of dispersion medium $(\mathrm{K}$ of water $=80$ ).

$\mathrm{X}=$ Potential drop in volts per $\mathrm{cm}$.

\section{Materials}

The vegetable tanning agents listed in Table I were kindly analyzed and supplied by. Mr. John Arthur Wilson.

For the electrophoresis measurements, the concentrations of tanning solutions were such that they contained 1 per cent tannin according to the above analyses, and the solutions were centrifuged for $5 \mathrm{~min}$. to remove coarse suspended matter. The tannin percentages were determined by the A. L. C. A, official method. They are merely arbitrary values which are used for purposes of commercial evaluation of vegetable extracts and, as shown by Wilson and Kern, ${ }^{10}$ do not represent the true values. When this work was commenced, however, they were the best figures available. Actually, with the exception of quebracho and osage orange, the solutions all contained about $4 \mathrm{~g}$. total solids per $100 \mathrm{cc}$.

\section{Results}

All the experimental data, accumulated over a 2-yr. period, cannot be recorded here. The following results are typical:

8 Phil. Mag., 1888, 60.

Ibid., 11 (1906), 425, 472.

${ }^{10}$ Thrs Journal, 12 (1920), 465.

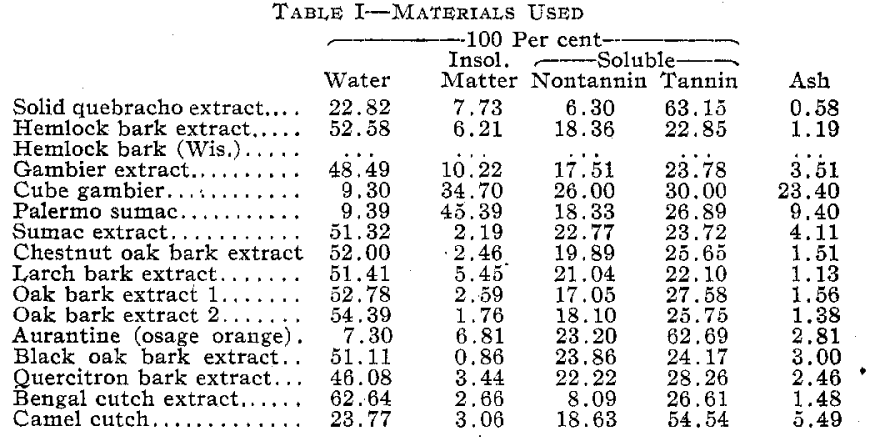

TABLE II-El-Ectrophoresis of CuBe Gambier

$4 \mathrm{~g}$, dry gambier extract per $100 \mathrm{cc}$; both electrodes set at 5 -cm. mark Levels of Gambier Solution Left Limb Right Limb

$\begin{array}{cccc}\text { Anode } & \begin{array}{c}\text { Right Limb } \\ \text { Cathode }\end{array} & \text { Time } & \text { Displacement } \\ \text { Cm. } & \text { Cm. } & \text { Min. } & \text { Cm. } \\ 7.8 & 8.0 & 0 & \text { Up } 0.2\end{array}$

$\begin{array}{llcc}7.8 & 8.0 & 0 & \mathrm{Up} 0.2 \\ 7.6 & 8.35 & 20 & \end{array}$

Av. 0.275 ith 1200 sec.

Average voltage through $19.9 \mathrm{~cm} .=114.3$

$\therefore$ Potential gradient $=5.74$ volts per cm.

1 Negative because particles migrated to anode.

TABLE III-ELECTROPHORESIS OF QUEBRACHO

$4 \mathrm{~g}$. dry quebracho per $250 \mathrm{cc}$; both electrodes at 5-cm. mark

Levels of Quebracho Solution

Left Iimb Right Iimb

Anode Cathode Time Displacement

$\begin{array}{llcc}\mathrm{Cm} . & \mathrm{Cm} . & \text { Min. } & \mathrm{Cm} . \\ 7.8 & 7.75 & 0 & \text { Up } 1.40\end{array}$

$\begin{array}{rrr}0.4 & 0 & \text { Up } 1.40 \\ 9.1 & 20 & \text { Down } 1.30\end{array}$

Av. 1.37 in $1200 \mathrm{sec}$.

Average voltage through $19.9 \mathrm{~cm} .=116.2$

$\therefore$ Potential gradient $=5.83$ volts per cm.

It will be noted in the electrophoresis of gambier that the displacement downward at the negative pole was greater than the upward movement toward the positive pole. This phenomenon is noted in the electrophoresis of all colloids. Burton referred to this effect in the electrophoresis of inorganic colloids as the "settling of the colloid." It is an error,

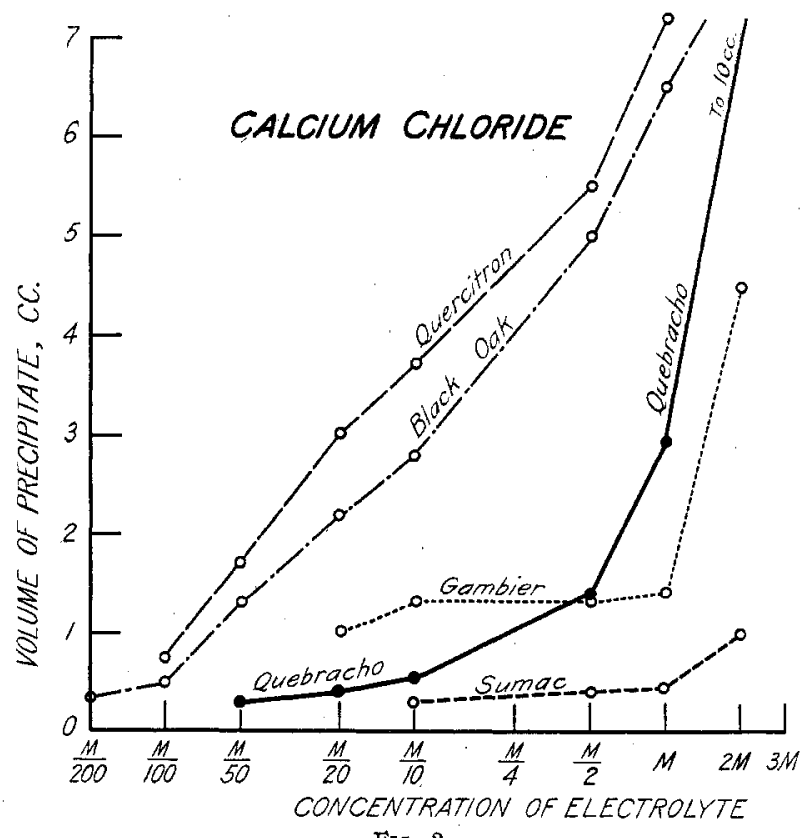

Fig, 2

however, that cannot be lightly turned aside. Unpublished results from this laboratory on the electrophoresis of iron oxide hydrosols show a downward displacement as much as 
five to ten times as great as the upward displacement, depending on the concentration of the hydrosol. It is evident that this effect throws great doubt upon all the absolute

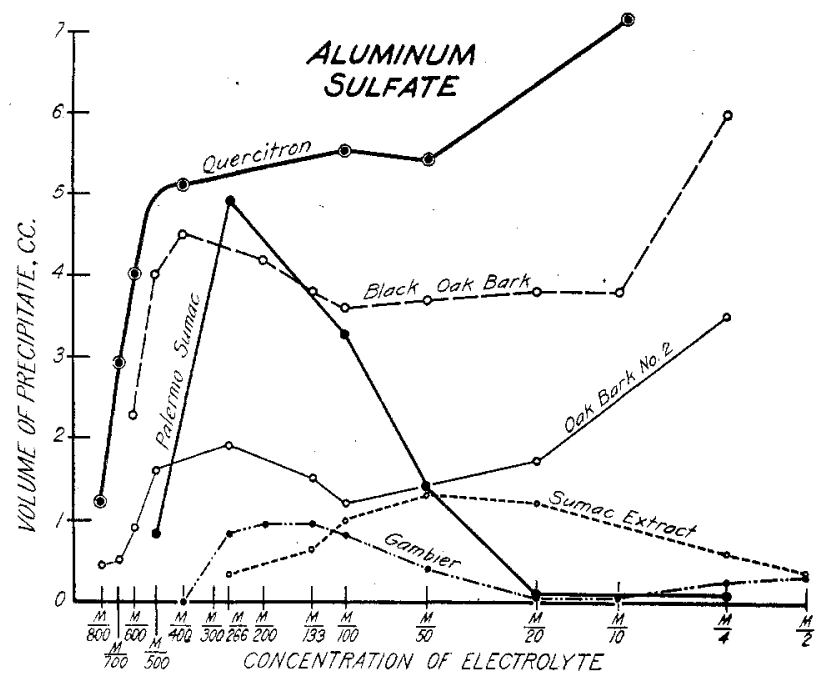

FIG. 3

values of electrical charge of colloids that are found in the literature. In a recent article, Burton ${ }^{11}$ promises an investigation of this "settling," which he attributes to the action of gravity accelerating the downward electrophoresis and retarding the upward movement.

The slightly greater upward movement noted in the particular quebracho experiment recorded was obtained with a number of extracts, and is probably due to a reprecipitation at the anode of some of the constituents of the liquor which were dissolved by the cathodic reaction in that limb of the $\dot{U}$-tube during the preliminary 5 -min. passage of the current in the reverse direction.

As a result of the effects of oxidation and reduction, as well as of formation of acid and alkali in the cathode and anode limbs, with the resulting dissolution and reprecipitation of some of the constituents of the tanning extract solutions, it was not always easy to tell just what was the electrophoresis displacement. The authors were obliged to make many preliminary experiments before they felt that they had one whose value they would care to publish. The behavior was not alike for any two extracts.

It is evident that the values which are given below for the electrical charges of the tannin particles are not absolutely correct. They are, however, not much more doubtful than the many published values obtained by electrophoresis. The authors do not attempt to calculate the values of the electrical charges per colloidal particle because such calculation involves the sizes of the particles. It is impossible to determine the sizes of colloidal particles by any method known at the present time and we are obliged to be content with values for the potential difference of the Helmholtz double layer only. The authors wish to emphasize the point that the published values for absolute electrical charges on colloidal particles are worthless, on account of the lack of a means for measurement of the sizes of colloidal particles.

RELATION BETWEEN P. D. AND ORDER OF CONDUCTIVITIESThe values obtained for the potential differences of the particles against the aqueous phase are listed in Table IV, together with the order of the conductivities. All the extracts showed anodic migration. The concentration in all the solutions was such as to give 1 per cent tannin, which

11 Proc. Roy. Soc. London, 95A (1919), 480. meant about $4 \mathrm{~g}$. total solids per $100 \mathrm{cc}$., except in cases of quebracho (2 g. per $125 \mathrm{cc}$.) and osage orange $(1.6 \mathrm{~g}$. per 100 cc.).

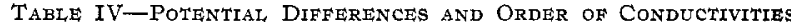

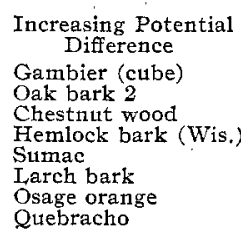

$\begin{array}{cl}\text { Volt } & \begin{array}{c}\text { Decreasing } \\ \text { Conductivity }\end{array} \\ -0.005 & \text { Sumac } \\ -0.009 & \text { Gambier } \\ -0.009 & \text { Oak bark } \\ -0.010 & \text { Larch bark } \\ -0.014 & \text { Hemlock bark } \\ -0.018 & \text { Chestnut wood } \\ -0.018(?) & \text { Osage orange } \\ -0.028 & \text { Quebracho }\end{array}$

The value for osage orange is the only one concerning which there is any great uncertainty.

According to the theory that the combination of tannin with hide substance is a function of the electrical charge of the particles, the most drastically acting extract would be quebracho, and the mildest would be gambier, according to the above values of the P. D.

By comparison with the conductivity series, it is evident that while, in general, the more highly conducting extracts have the lower $P$. D. values, there are exceptions to this rule, so that it is apparent that the magnitude of the electrical charge is a function of the kind as well as the amount of nontannins present, as is to be expected.

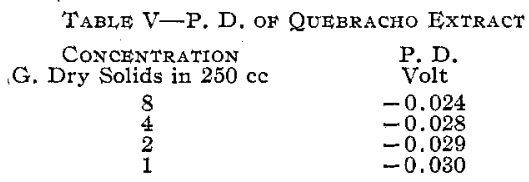

RELATION BETWEEN P. D. AND CONCENTRATION-The results obtained upon measuring the $\mathrm{P} . \mathrm{D}$. of a quebracho extract at different concentrations are assembled in Table $\mathrm{V}$.

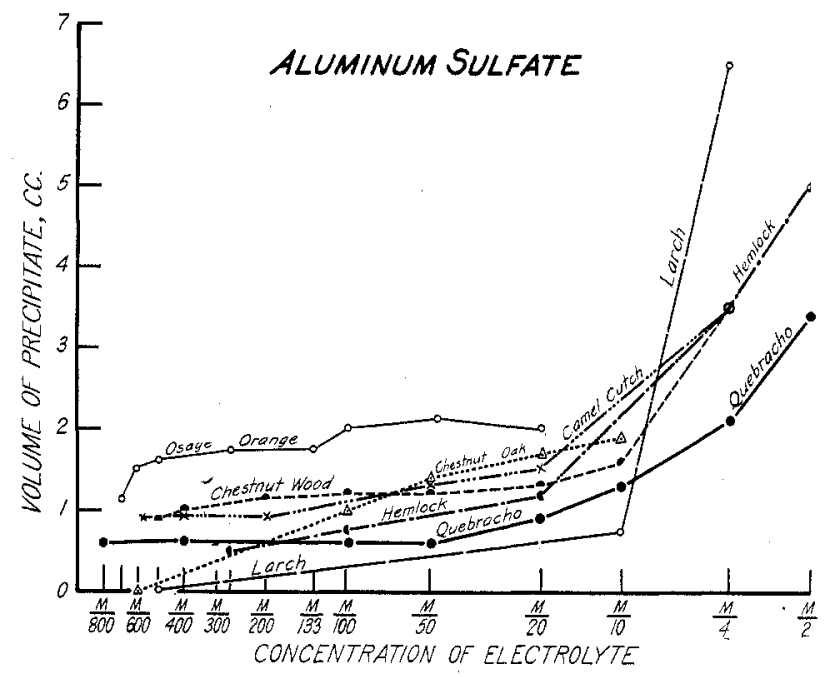

FIs. 4

The P. D. increases upon dilution of the extract, as is to be expected according to the "complex theory" of colloids, since dilution decreases the concentration of electrolyte in the outer layer surrounding the particle and permits greater ionization of the peptizing electrolyte adsorbed on the particle.

RELATION BETWEEN P. D. AND ACIDITY-Since the negative charge on a colloid is readily increased by the addition of hydrogen ion, such addition ought to lower the P. D. of a vegetable tanning extract. The data in Table VI show that this is true. 

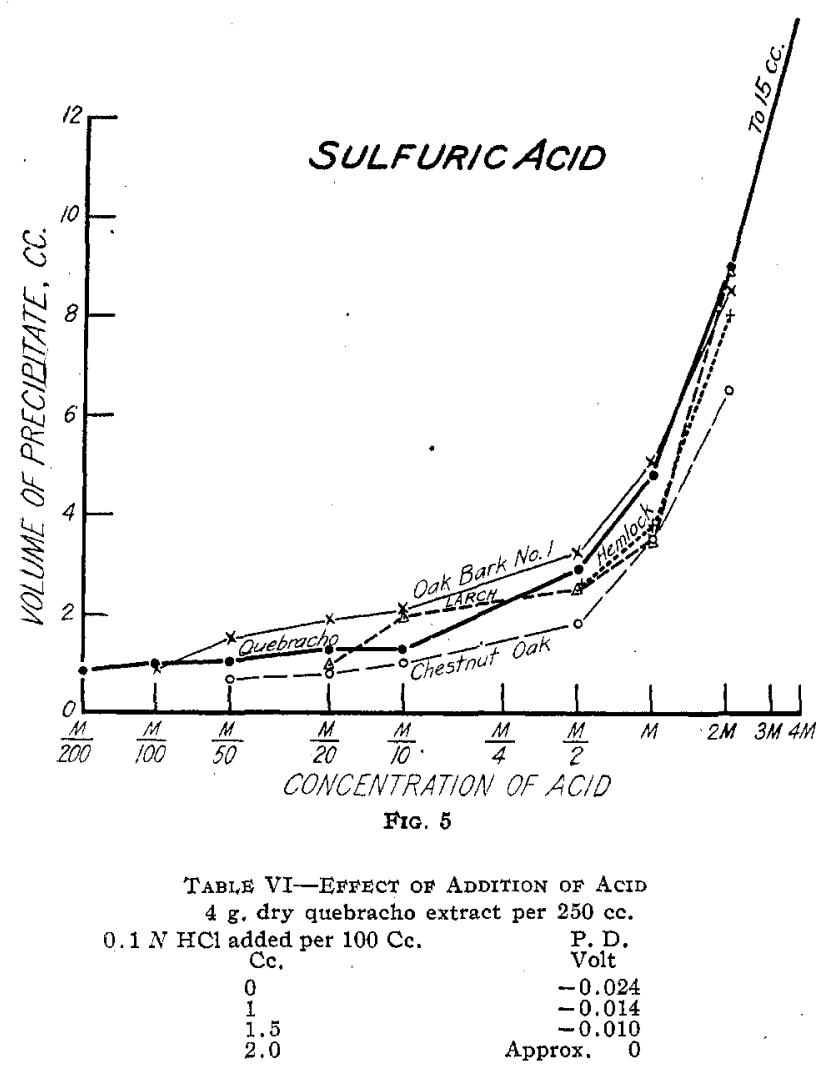

EFFECT OF DIALYSIS-On the other hand, dialysis of extracts ought to raise the $P$. D., as a result of the removal of nontannins from the solution. Table VII describes the results obtained by dialysis through collodion sacs against distilled water.

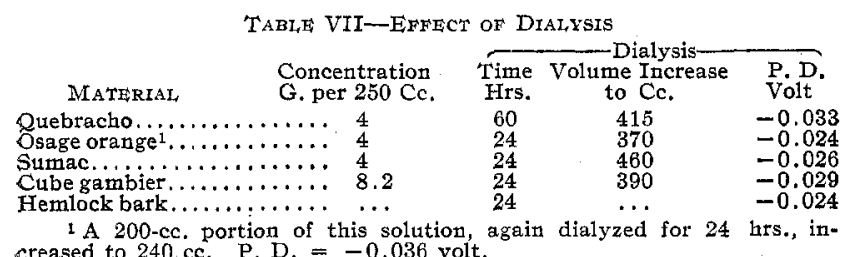

In all cases the P. D. increased as was expected. It is true that part of the increase in P. D., is attributable to the dilution of the solution, but the removal of the nontannins also was a contributing factor.

\section{PRECIPITATION OF VHGETABLE TANNING EXTRACTS}

The action of vegetable tanning extracts toward electrolytes should throw some light upon their colloidal nature. With this idea in mind many extracts were treated with various electrolytes in different concentrations and the volumes of precipitated material were measured.

\section{TeChnto}

Aqueous dispersions or solutions of the extracts were made up so that $100 \mathrm{cc}$. of solution contained $4 \mathrm{~g}$. of total solids. Before adjusting to the mark, the solutions were kept at $85^{\circ} \mathrm{C}$., and cooled to $25^{\circ} \mathrm{C}$. before adjusting to volume. The stock solution was then centrifuged for 5 min. at about 1000 "times gravity" in order to throw down coarse suspended matter.

Twenty-five-cc. portions were placed in 100-cc. graduated "oil tubes" (tubes tapering to a conical end). Equal volumes of electrolyte solutions of varying concentration were poured into each tube, allowed to stand for 15 to $30 \mathrm{~min}$, since precipitation was not always instantaneous, and finally centrifuged for 5 min. at 1000 "times gravity." The volumes of the precipitates were plotted against the concentrations of electrolyte employed.

\section{Results}

The results may be most conveniently studied by grouping them under the various electrolytes used. Each available extract was not tested with all electrolytes since in some cases preliminary experiments indicated that further work would be fruitless.

Monovalent CATION. Potassium Chloride-Concentrations of potassium chloride from $0.02 M$ to $4 M$ gave only negligible amounts of precipitate with gambier and quebracho. Oak bark gave a gradually increasing salting out effect. Since gambier and quebracho represent extreme types of tans no further tests were made with this salt.

Hydrochloric Acid-Concentrations from $0.01 M$ to 6 $M$ were used. Gambier and quebracho showed appreciable amounts of precipitate only at the highest concentration of the acid and since this was not a simple colloid precipitation, no further experiments were attempted. (Fig. 6.)

Sulfuric Acid-Quebracho, hemlock, oak bark, chestnut oak, and gambier gave progressively increasing amounts of precipitate (salting out) with concentrations of acid from $0.005 M$ to $4 M$. (Fig. 5.) No precipitate was obtained with sumac until molal acid was reached. Acid of this concentration threw down gummy masses, similar to those obtained with aluminium sulfate. At $4 M$ concentration a flocculent precipitate was formed.

Phosphoric Acid-Gambier began to give an appreciable precipitate only at 4 to $7 M$ concentration. With sumac a gummy mass was thrown out at $2 M$, as was observed in the case of sulfuric acid and aluminium sulfate, and at $4 M$ to $7 M$ a flocculent precipitate formed which left the supernatant solution almost colorless. A salting out effect was obtained with quebracho. (Fig. 6.)

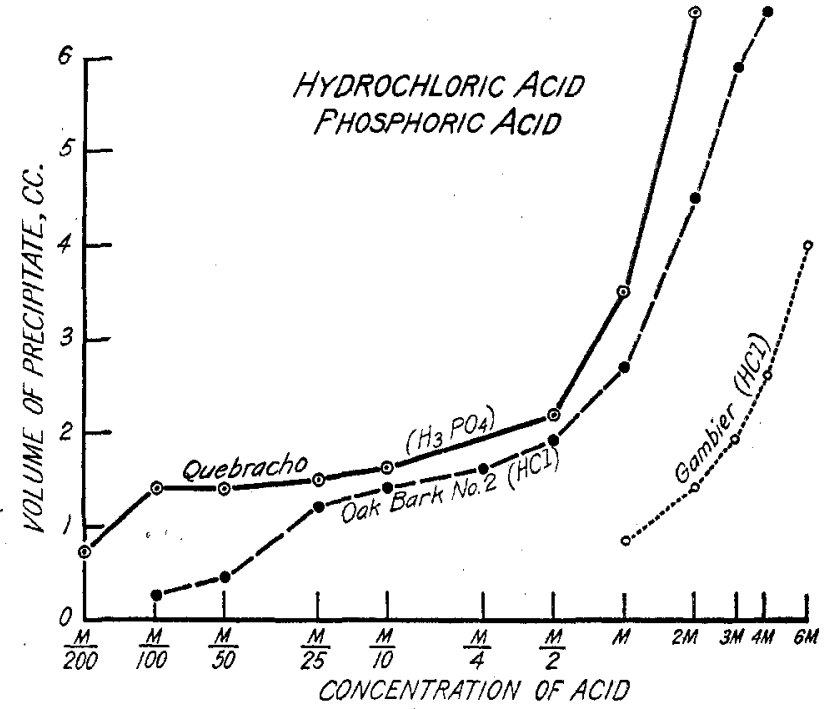

FIG. 6

Acetic Acid-Experiments with quebracho, sumac, gambier, and oak bark were run with concentrations of acid from $0.005 M$ to $4 M$. There was no appreciable precipitation in any case. It is of interest to note that at the higher concentrations the suspended matter began to dissolve or peptize.

Formic Acid-Concentrations from $0.005 M$ to $12.5 \mathrm{M}$ were used. Sumac, chestnut oak, hemlock, gambier, larch 
bark and oak bark gave no precipitation up to $4 M$. The suspended matter began to dissolve or peptize at $4 M$. Quebracho and quercitron were precipitated, but the precipitate dissolved at $2 M$ to $4 M$. (Fig. 7.)

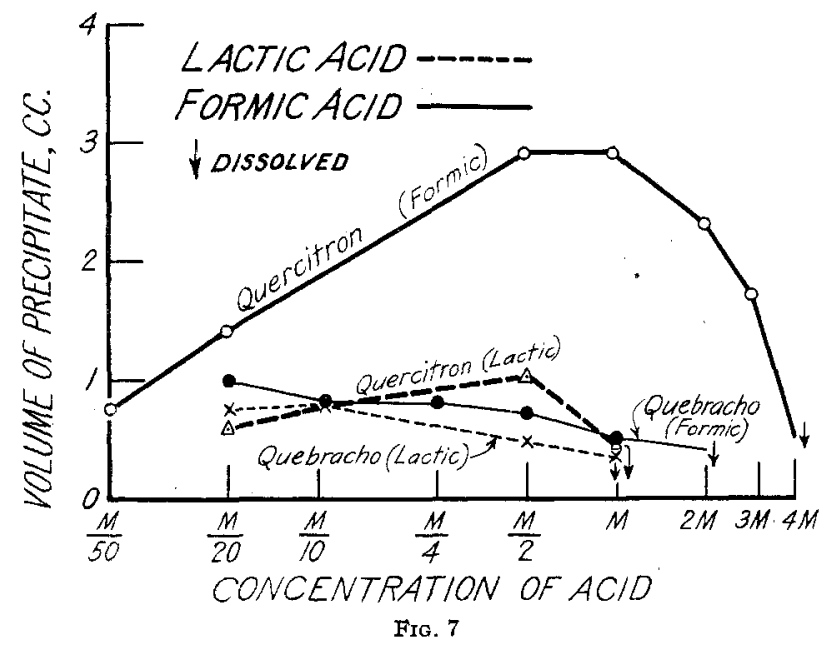

Lactic Acid-Concentrations from $0.005 \mathrm{M}$ to $2 \mathrm{M}$ were employed. The effects of this acid were similar in kind, but not in degree, to those with formic acid. (Fig. 7.) The precipitates with quercitron and quebracho redissolved at lower concentrations of lactic acid than of formic acid. Since lactic is the weaker acid, and also since this redissolving was not found with hydrochloric or with sulfuric acids, the phenomenon must be due to chemical properties other than concentration of hydrogen ion.

DIVALENT CATIONS. Barium Chloride-On account of limitations of solubility, this salt was employed up to a final concentration of $0.5 \mathrm{M}$. Progressive salting out occurred with oak bark, chestnut wood, quebracho, gambier, and hemlock. (Fig. 1.)

Calcium Chloride-Final concentration of $2 M$ was possible with this salt. As with barium chloride, increasing amounts of precipitate were obtained with quercitron, black oak, quebracho, gambier, and sumac. At the same concentration of these salts the different extracts gave in some cases less, and in others more, precipitate, showing the presence of substances reacting with barium and calcium ions to form chemical compounds of different solubilities. (Fig. 2.)

TRIVALENT CATION. Aluminium Sulfate-In the precipitation of negatively charged colloidal dispersions, aluminium sulfate is not only a powerful precipitant, but it also gives the "irregular series" or "tolerance zone" which is typical of the action of weak base cation, strong acid anion salts, as shown by Buxton and Teague, 12 and by Freundlich and Schucht. ${ }^{13}$

The concentrations of aluminium sulfate used ranged from $0.00125 \mathrm{M}$ to $0.5 \mathrm{M}$.

The "irregular series" effect was obtained with the following extracts: gambier, sumac, Palermo sumac, oak bark No. 2, black oak bark, and not so markedly with quercitron. Precipitation set in generally at $0.00125 M$ concentration of the salt, rose rapidly to a maximum, dropped off into a "tolerance zone," and then started upward again. (Fig. 3.)

Those which gave no "irregular series," at least up to $0.5 M$ concentration of the salt, were: Osage orange, chestnut wood, chestnut oak, hemlock bark, larch bark, quebracho and camel cutch. They began to precipitate at $0.00125 M$, rising gradually to about $0.1 M$ where there

\footnotetext{
12 Z. physik. Chem., 57 (1907), 76.
}

13 Ibid., 85 (1913), 641 . was an abrupt upward trend similar to a salting-out effect. These extracts are not so sensitive to precipitation by aluminium sulfate as the first mentioned.

Bengal cutch is in a separate category, since it was unaffected by the addition of aluminium sulfate.

\section{INTERPRETATION OF RESUITS}

The interpretation of the effects of the several electrolytes is masked by the salting out and chemical precipitation of the molecularly dispersed substances present in addition to the colloidal tannins. The writers had hoped - that the order of sensitiveness to precipitation by mono-, di-, and trivalent cations would help show the order of magnitude of the potential differences of the particles against the aqueous phase, but the interference by the large amounts of molecular dispersions of nontannins in salting out spoiled this anticipation. The presence of this salted-out, molecularly dispersed material was shown in several cases where the coagula caused by the calcium and aluminium salts were found to redissolve to a large extent upon addition of water. If it were all gel substance it should not be reversible.

The conduct of the extracts shows that there is a large amount of colloidal matter present, that it belongs to a type of dispersion with properties between the intermediate and hydrophilic dispersions, and, hence, that the measurements reported in the first part of the paper are justified.

From the colloidal point of view, vegetable tanning extracts constitute an unexplored field, and the authors believe that the precipitation graphs included in this article will prove of value when interpreted in the light of further research.

\section{ACKNOWLEDGMENT}

We take pleasure in expressing our indebtedness to Messrs. A. F. Gallun and Sons Co., of Milwaukee, for grants in aid of this investigation.

\section{National Safety Council Census}

It is known that the chemical industry, because of its inherent hazards, has always been among the leaders in industrial safety, but it has never been definitely established how many persons in the chemical industry are engaged in accident prevention and industrial health work. The National Safety Council is now taking a census of safety men in the chemical industry, as well as in all other industries and in public safety work.

When this census is completed it will give a good indication of the extent of such work in chemical plants all over the country, and will afford the first basis of comparison with similat work in other industries. The census will include not only members and employees of members of the National Safety Council, but all other persons engaged in safety and industrial health activities. Most of the principal chemical plants are members of the Coun-, cil. The headquarters of the Council are at $168 \mathrm{~N}$. Michigan Ave., Chicago, I11.

\section{Associated Technical Societies of Detroit}

The Associated Technical Societies of Detroit, an affiliation of the architectural, engineering, and other technical societies of the city, has just been organized. The Detroit Section of the American Chemical Society is one of the members of the association.

The paramount use of the association to its members and to the public is its opportunity for public service for the city and for the state of Michigan. It will take an active interest in all matters where engineering, architecture, or technical subjects are of importance. The council will assist in furnishing definite and accurate information to the public. 'The association will offer its assistance and advice to city and state officials whenever required. The purpose of the association's activities is to provide the most reliable technical information for the proper consideration of public improvements and public undertakings. 